\title{
JPM-4 Proton Precession Magnetometer and Sensitivity Estimation
}

\author{
Xiaorong Gong, ${ }^{1}$ Shudong Chen, ${ }^{1}$ Shuang Zhang, ${ }^{1 *}$ and Xin Guo ${ }^{2}$ \\ ${ }^{1}$ College of Electronic Science and Engineering, Jilin University, Changchun 130012, China \\ ${ }^{2}$ College of Physics, Jilin University, Changchun 130012, China
}

(Received November 1, 2021; accepted December 10, 2021)

Keywords: sensitivity, proton magnetometer, scale quantum magnetometer, Larmor precession, magnetic sensors

A proton precession magnetometer (PPM) is a traditional quantum magnetometer based on the Larmor precession of hydrogen protons in Earth's magnetic field. PPMs are widely used in various fields, such as magnetic observation and detection of buried objects. A coaxial solenoid is typically used to construct the PPM sensor. However, the Larmor signal slowly weakens when the axial direction of the sensor gradually approaches the direction of Earth's magnetic field. Thus, a dead zone exists when Earth's magnetic field is nearly parallel to the axis of the sensor. An omnidirectional sensor with an " 8 "-type structure is designed in this study. The signal quality is slightly affected by the orientation of the sensor owing to the orthogonal polarized magnetic field components. The measured signal-to-noise ratio (SNR) of the Larmor signal is approximately $31 / 1$, and the decay constant of the free induced decay (FID) signal is $0.95 \mathrm{~s}$. The electrical parameters of the sensor coil are optimized and the polarization power is $8.0 \mathrm{~W}$. Multiple hourly observations of Earth's magnetic field in noisy and quiet environments indicate the satisfactory consistency of the measurement results of the two PPMs with the proposed sensor. The standard deviations (STDs) of the measured results for a single instrument in noisy and quiet environments are 6.4 and $0.076 \mathrm{nT}$, respectively, which effectively reflect the environmental noise level. The sensitivity of the instrument is estimated to be $0.04 \mathrm{nT}$ at a $5 \mathrm{~s}$ cycling rate for the two synchronized instruments. This is higher than the sensitivity of most commercial magnetometers of $0.1 \mathrm{nT}$.

\section{Introduction}

A proton magnetometer is a scalar quantum magnetometer based on nuclear magnetic resonance (NMR) for the measurement of the total magnetic field. NMR studies from the 1950s and 1960s laid the theoretical foundation for the implementation of proton magnetometers. ${ }^{(1,2)}$ Proton magnetometers include two types: one is the classical proton precession magnetometer (PPM), which was first implemented by Varian and Waters; ${ }^{(3-5)}$ the other is the Overhauser magnetometer (OVM), which is based on Overhauser-effect-enhanced NMR. ${ }^{(6-9)}$ A PPM exploits a DC-generated magnetic field to polarize the hydrogen protons. The proton magnetic

*Corresponding author: e-mail: 1786099678@qq.com

https://doi.org/10.18494/SAM3719 
moment rotates around the external magnetic field $B_{0}$ with angular velocity $\omega_{0}$ after quickly removing the polarized field, and $B_{0}$ can be obtained by the accurate calculation of $\omega_{0}$ using the equation $\omega_{0}=\gamma_{p} B_{0} \cdot{ }^{(10)}$ An OVM employs radio frequency to resonate electrons, and the resonated electrons polarize hydrogen protons, resulting in enhanced proton polarization. ${ }^{(11-13)}$ Although OVMs have replaced classical PPMs in aerospace and satellite magnetic measurement applications owing to their advantages of a high cycling rate and sensitivity and low power consumption, classical PPMs are still widely used in mineral exploration, geological mapping, buried or sunken object detection, magnetic observatory use, archaeological prospecting, and ionospheric research due to their low cost, easy production, long life, and high stability. ${ }^{(10,14)}$

PPMs can obtain strong Larmor signals by enhancing polarization fields, thus increasing their sensitivity. Reports indicate that the power consumption of PM7 can reach $15 \mathrm{~W} .^{(15)}$ However, the cost of enhanced polarization is increased power consumption, which leads to additional Joule heat loss and coil heating, resulting in adverse temperature effects. In addition, high power consumption means frequent charging and reduced portability. Therefore, obtaining high sensitivity at a low power loss is the first consideration in PPM design. Optimized coil parameters are used in this study to reduce the power consumption substantially.

A coaxial solenoid coil has a simple structure and is easy to wind. This coil is the structure often used by PPM sensors and the sensor coil of OVM sensors. ${ }^{(10,16)}$ However, a sensor of the polarized magnetic field possesses a dead zone when the external magnetic field is nearly parallel to the axis. A structure of two inverted side-by-side solenoids that improved the omnidirectionality of the sensor has been presented. ${ }^{(15)}$ However, the disadvantage of this structure is its large coil volume, which degrades the gradient tolerance of the sensor. In this study, a compact sensor design, which significantly reduces the size of the sensor and enhances the omnidirectional characteristics, is presented considering these defects.

The technical specifications of a PPM include sensitivity, (absolute) accuracy, measurement range, gradient tolerance, cycling rate, drift, temperature range, and power consumption. Among these specifications, sensitivity is one of the most important technical specifications. We believe that studies on the evaluation methods of technical specifications of both PPMs and OVMs have been insufficient. Previous studies mainly evaluated sensitivity without considering the impact of electromagnetic interference (EMI) noise, thus resulting in inconsistencies between the estimated and actual sensitivity values. ${ }^{(16-19)}$ The EMI component is usually larger than the reading noise of the instrument. Thus, it is not possible to accurately obtain the sensitivity unless environmental interference is eliminated. To this end, a synchronization function with a synchronization error of less than $1 \mathrm{~ms}$ is added to the JPM-4 magnetometer to ensure the simultaneous measurement by two JPM-4 magnetometers, and the EMI can be eliminated by taking the difference between the measurement results of the two instruments. In addition, a method using an equal amplitude signal generated by the signal source to evaluate the sensitivity has recently been reported. The signal-to-noise ratio (SNR) of the signal generated by the signal source is considerably higher than that of the Larmor signal of the magnetometer, thus also leading to incorrect estimations of sensitivity. ${ }^{(18)}$

The JPM-4 proton magnetometer described in this paper was designed by Jilin University of China. The JPM-4 magnetometer, including the sensor and its analog and digital parts, is 
comprehensively introduced in this paper. Its sensitivity was measured synchronously by two instruments in an outdoor environment, and environmental noise was evaluated by a single instrument. Experimental results indicate that the sensitivity estimation method proposed in this paper is helpful for developers and users to test and evaluate instruments conveniently. Moreover, the results reveal that the instrument has sufficiently high sensitivity to observe Earth's magnetic field.

\section{Physical Principles}

Subatomic particles such as protons possess an angular momentum $\boldsymbol{L}$ and a magnetic moment $\boldsymbol{\mu}$ due to the spin of the hydrogen nucleus. The relationship between $\boldsymbol{L}$ and $\boldsymbol{\mu}$ is

$$
\boldsymbol{\mu}=\gamma p \boldsymbol{L},
$$

where $\gamma_{p}$ is the gyromagnetic ratio, which is associated with the charge-mass ratio. The magnetic moment $\boldsymbol{\mu}$ has a moment $\boldsymbol{T}$ under the action of the external magnetic field $\boldsymbol{B}$, and $\boldsymbol{T}$ forces $\boldsymbol{\mu}$ around $\boldsymbol{B}$ with angular velocity $\boldsymbol{\omega}$ :

$$
\boldsymbol{T}=\boldsymbol{\mu} \times \boldsymbol{B} .
$$

Using $\dot{\boldsymbol{L}}=\boldsymbol{T}$, we obtain

$$
\boldsymbol{\omega}=\gamma_{p} \boldsymbol{B} .
$$

The total proton magnetic moment rotates around the $\boldsymbol{B}_{\boldsymbol{p}}$ axis when a polarized magnetic field $\boldsymbol{B}_{\boldsymbol{p}}\left(\boldsymbol{B}_{\boldsymbol{p}}>>\boldsymbol{B}\right)$ is applied, and this process is called the longitudinal relaxation process. By contrast, the total magnetic moment rotates around the $\operatorname{target} \boldsymbol{B}$ with angular velocity $\boldsymbol{\omega}$ upon the sudden removal of $\boldsymbol{B}_{p}$, and this process is called the transverse relaxation process. Finally, $\boldsymbol{B}$ can be calculated from the measured $\omega$ using Eq. (3).

\section{Implementation of JPM-4 PPM}

\subsection{Brief introduction to JPM-4 PPM}

As shown in Fig. 1, the JPM-4 proton magnetometer comprises two parts: the sensor and the instrument console. The console includes four parts: digital board [Fig. 2(a)], analog board [Fig. 2(b)], key display board, and power board.

A block diagram of the JPM-4 proton magnetometer is shown in Fig. 3. Figure 3 shows that an Advanced RISC Machine (ARM) controls the complex programmable logic device (CPLD) to perform polarization and tuning. The sensor coil and tuning capacitor constitute an LC parallel resonance circuit. The resonance-amplified signal is sent to the amplifier to be intensified to the order of volts. The amplified signal is sent to the comparator, where it is shaped into a square 


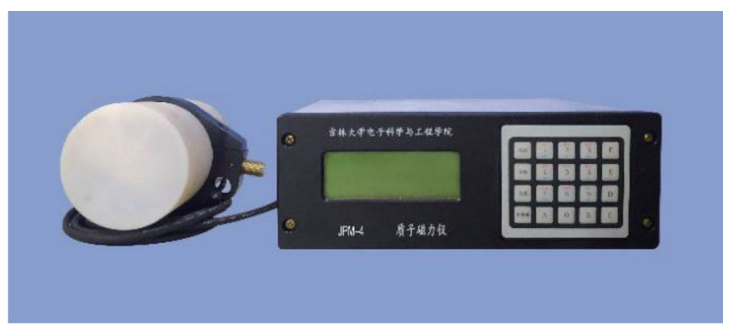

Fig. 1. (Color online) JPM-4 proton magnetometer.

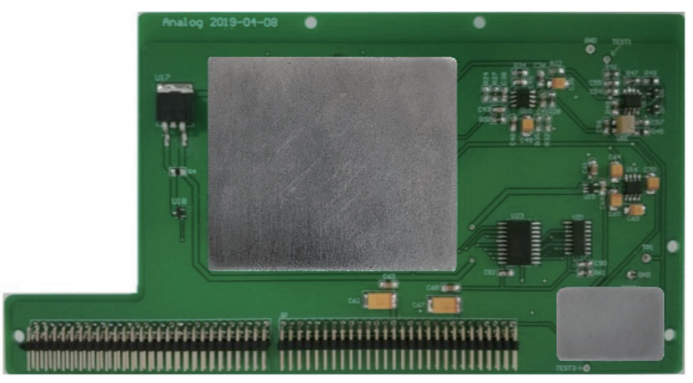

(a)

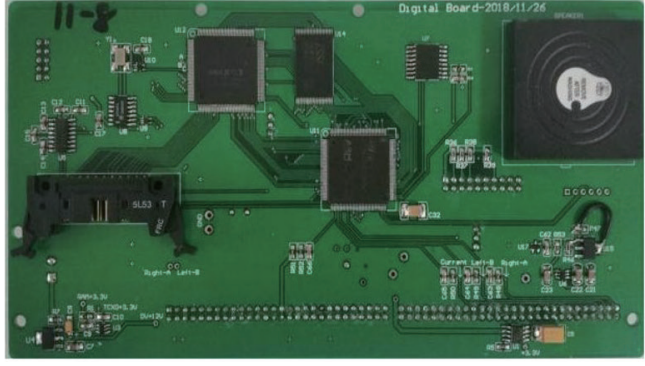

(b)

Fig. 2. (Color online) (a) Analog board and (b) digital board of the JPM-4 proton magnetometer.

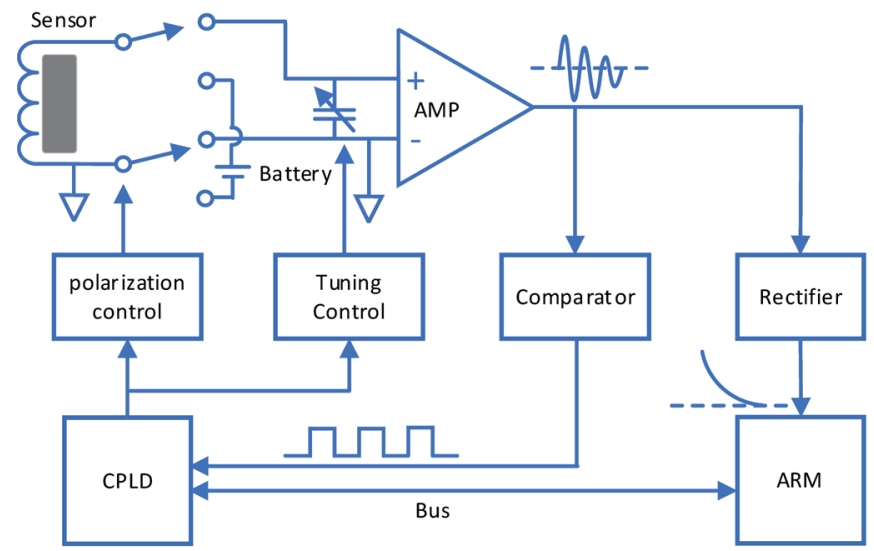

Fig. 3. (Color online) Block diagram of the JPM-4 proton magnetometer.

wave, and then it is sent to the CPLD for counting. The count value is transmitted to the ARM to calculate the magnetic field. The rectifier aims to obtain the envelope of the signal, which helps the ARM monitor the signal quality.

\subsection{Sensor design}

Solenoidal, toroidal, and cylindrical sensor coils are respectively presented in Figs. 4(a)4(c). ${ }^{(10)}$ The corresponding polarization fields simulated by MAXWELL software are shown in 


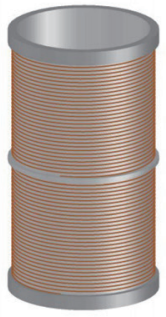

(a)

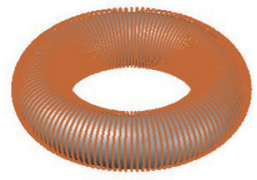

(b)

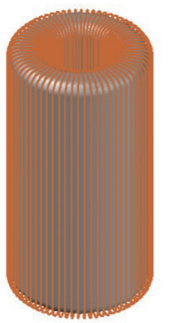

(c)

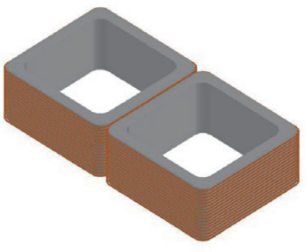

(d)

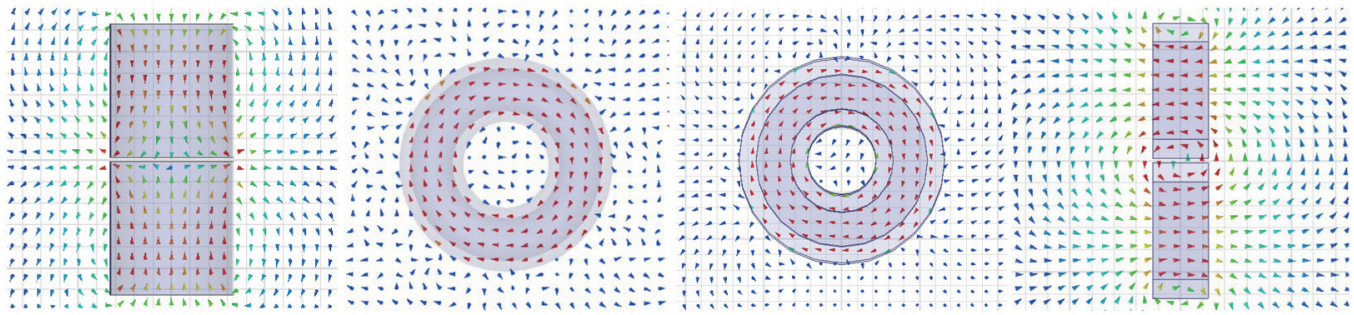

(e)

(f)

(g)

(h)

Fig. 4. (Color online) Simulation of four typical proton sensor structures and their internal polarized magnetic field distributions. (a, e) Solenoidal coil, (b, f) toroidal coil, (c, g) cylindrical coil, and (d, h) " 8 " type coil.

Figs. 4(e)-4(g), respectively. The polarized magnetic fields generated by the toroidal and cylindrical coils have a circular magnetic field with two orthogonal components. Therefore, toroidal and cylindrical coils have good common-mode anti-interference capability and an omnidirectional characteristic. However, these coils are difficult to wind. A solenoid can only produce a polarized magnetic field in one direction despite its easy winding. The induced precession signal disappears when the surrounding magnetic field is along the axis of the sensor due to zero magnetic torque. Figures 4(d) and 4(h) show the sensor constructed in this study, which is a side-by-side solenoidal configuration named an " 8 "-type coil, and its polarization field, respectively. Figure 5 shows an image of the 8 type coil; two sets of coils are wound in reverse directions to eliminate common-mode interference, thus substantially reducing the induced external EMI noise. ${ }^{(16)}$ The 8-type coil improves the directivity by equalizing the two orthogonal polarized magnetic field components. The coil also has good directivity for its orthogonal magnetic field.

The low-frequency coil is used for DC polarization and free induced decay (FID) signal reception. The coil parameters should provide the best performance for polarization and reception. Comprehensive consideration of the size, power consumption, and SNR yields the following values: the resultant number of turns is 530 , the wire gauge is $0.53 \mathrm{~mm}$, the inductance is $34 \mathrm{mH}$, the resistance is $18 \Omega$, and the resonant frequency is $30 \mathrm{kHz}$ after shielding.

\subsection{Analog circuit designs}

Figure 6 shows that the analog circuit comprises seven sections: polarization circuit, tuning circuit, pre-amplifier, amplifier, bandpass filter, comparator, and rectifier. The polarization 


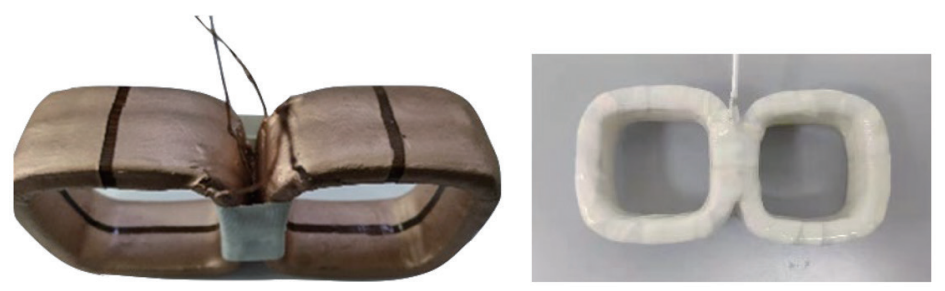

Fig. 5. (Color online) Image of 8 type proton sensor coil with electrostatic shielding.

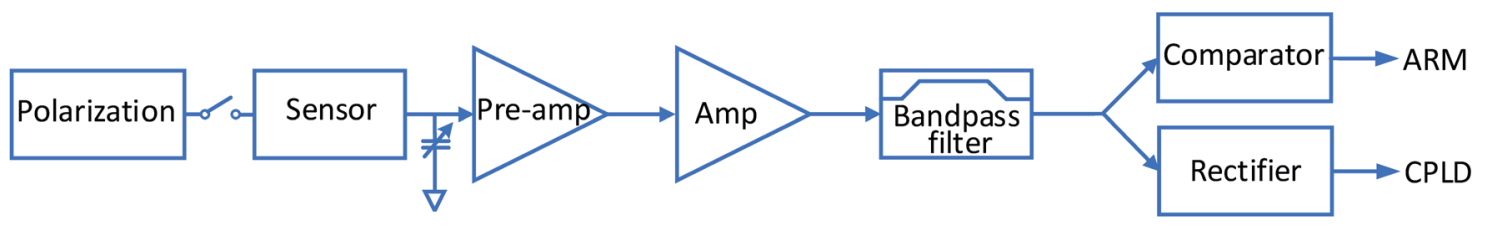

Fig. 6. (Color online) Block diagram of analog board.

circuit includes a switch to shift between polarization and reception, which are both controlled by the CPLD. The polarization time is set to $1.6 \mathrm{~s}$ and the receiving time is set to $1.4 \mathrm{~s}$. This setting benefits from a short longitudinal relaxation time for kerosene and an acceptable lateral relaxation time. The key feature of the polarization circuit is that the polarization current is turned off quickly to avoid affecting the magnetic field to be measured. Figure 7 shows that the polarization circuit can turn off the polarization current within $400 \mu$ s to prevent the hydrogen proton magnetic moment from conducting Larmor precession around $\boldsymbol{B}_{\boldsymbol{p}}$ for a long time, decreasing the initial amplitude of the signal.

As shown in Fig. 8, a tuning capacitor is placed in parallel with the sensor coil to achieve a measurement range of 20-120 $\mu$ T. In Fig. 8, $L$ is the coil inductance, $r$ is the coil resistance, $C$ is the tuning capacitance, $L C$ is the parallel resonance applied to amplify the signal without reducing the SNR, and $R_{d}$ is the matching resistance operated in the underdamped state to limit the quality factor $Q_{0}$ to avoid circuit resonance. The quality factors without and with $R_{d}$ are respectively expressed as

$$
\begin{gathered}
Q_{0}=\frac{L \omega}{r}, \\
Q \approx \frac{R_{d}}{Q_{0}^{2} r+R_{d}} Q_{0} .
\end{gathered}
$$

The calculated $Q_{0}$ and $Q$ are 27 and 23, respectively.

The pre-amplifier is a low-noise amplifier JFET with a voltage noise of $1 \mathrm{nV} /(\mathrm{Hz})^{1 / 2}$ and a current noise of $0.1 \mathrm{pA} /(\mathrm{Hz})^{1 / 2}$ at $1 \mathrm{kHz}$. Resistors with high accuracy and temperature stability are used throughout the amplifier chain to reduce the addition of Johnson noise. Figure 9 shows that a $380 \mathrm{mV}$ sinusoidal signal generated by an AFG-2225 digital signal source is divided into $47 \mu \mathrm{V}$ by resistors $R_{1}=414 \mathrm{k} \Omega$ and $R_{2}=51 \Omega$. Our test simulated a Larmor input signal with an 


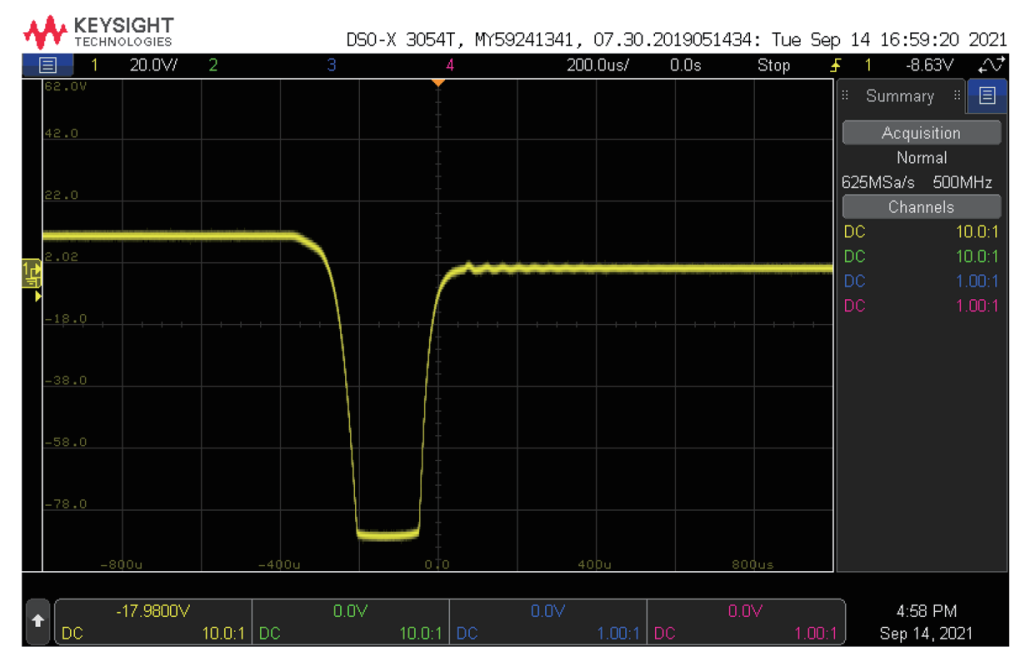

Fig. 7. (Color online) Reverse voltage of PMOS.

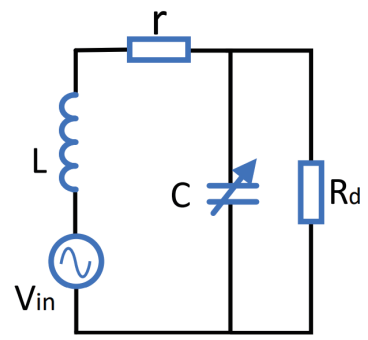

Fig. 8. (Color online) Effective circuit of sensor.

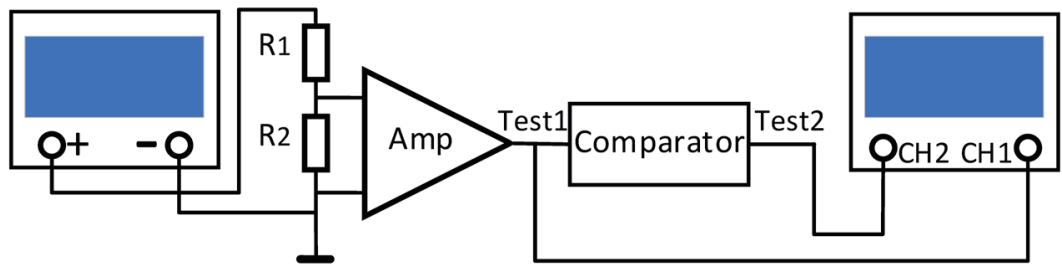

Fig. 9. (Color online) Schematic of amplifying signals and test of shaping the signal.

initial amplitude of $47 \mu \mathrm{V} / 23=2 \mu \mathrm{V}$. Figure 10 shows the amplified FID signal (yellow line) and square wave (green line) on a DSOX3504T oscilloscope after shaping by the comparator. The electromagnetic induction signal is proportional to the frequency $f$ and $Q$. Thus, a second-order low-pass filter with $850 \mathrm{~Hz}$ bandwidth is used to flatten the transmission characteristics between $850 \mathrm{~Hz}$ and $5.1 \mathrm{kHz}$.

Figure 11 shows the FID signal measured by a Rohde \& Schwarz RTH1002 digital oscilloscope in a field environment. The initial amplitude is $2.3 \mathrm{~V}$ and the decay constant is $0.95 \mathrm{~s}$. The standard deviation (STD) of the noise is measured as $75 \mathrm{mV}$ and the SNR is 2300/75 $=31$. 


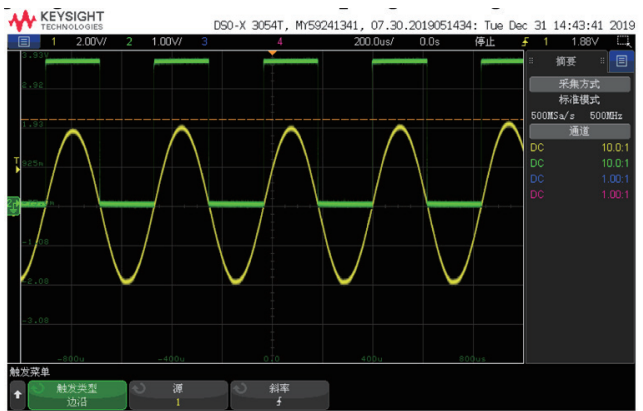

Fig. 10. (Color online) Simulated FID and shaping signals.

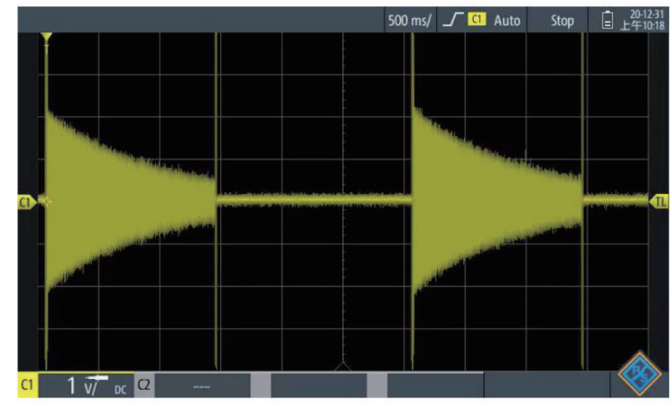

Fig. 11. (Color online) Measured FID signal.

\subsection{Digital board designs}

The low-power STM32 and CPLD are chosen to perform measurement control, data transmission, magnetic field calculation, storage, and other functions. Among these designs, the CPLD mainly performs measurement timing control, counting, and other functions. The FLASH storage space is $256 \mathrm{Mbit}$, which can store up to $500 \mathrm{k}$ magnetic field values and various initial values, such as measurement modes, to avoid information loss after shutdown. The magnetometer can transmit measurement data to a PC via RS232. The keyboard has $4 \times 5=20$ keys, while the LCD uses a $192 \times 64$ low-temperature liquid crystal.

The JPM-4 magnetometer has two modes: base station and mobile. This magnetometer has a settable measurement cycle time, real-time clock, automatic and manual tuning, and other functions. The mobile measurement mode has three coordinate systems: $X Y$, line station, and GPS. Real-time data can also be viewed. Two JPM-4 magnetometers can also perform highprecision time synchronization with a synchronization error of less than $1 \mathrm{~ms}$. The polarization and receiver control sequences are shown in Fig. 12. In these sequences, the polarization time is $T_{1}=1.6 \mathrm{~s}$ and the receiving time is $T_{2}=1.4 \mathrm{~s}$.

Figure 13 is a schematic of the frequency measurement. Figure 13(a) shows the counting command (high level) sent by the ARM to the CPLD through the data bus after the end of polarization, and the duration $T$ depends on the signal quality controlled by the ARM. $T x$ in Fig. 13(b) is the actual frequency measurement time of the CPLD. Figure 13(c) shows the square wave of the signal to be measured and Fig. 13(d) shows the reference signal of the temperaturecompensated X'tal (crystal) oscillator (TCXO). The frequency measurement is completed through cooperation between the ARM and the CPLD. The ARM is responsible for sending control instructions and for data calculation, while the CPLD is responsible for counting the rising edges of measured and reference signals, where the reference signal is provided by the $4 \mathrm{MHz}$ TCXO with 2 ppm accuracy.

In Fig. 13(c), $N$ is the number of cycles of the signal to be tested. The actual measurement time is $T x=N / f_{s}$, where $f_{s}$ is the frequency of the measured square wave. The measured signal frequency can be calculated as 


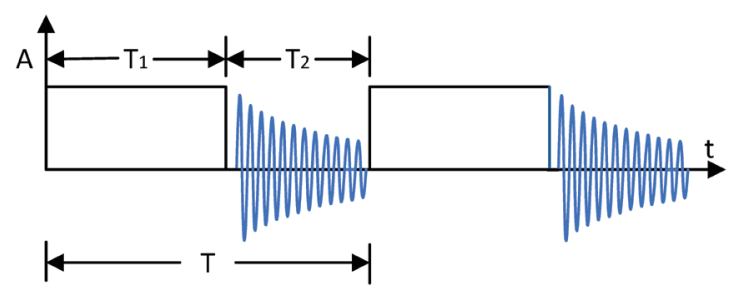

Fig. 12. (Color online) Sequences of polarization and reception controlled by the ARM and CPLD.

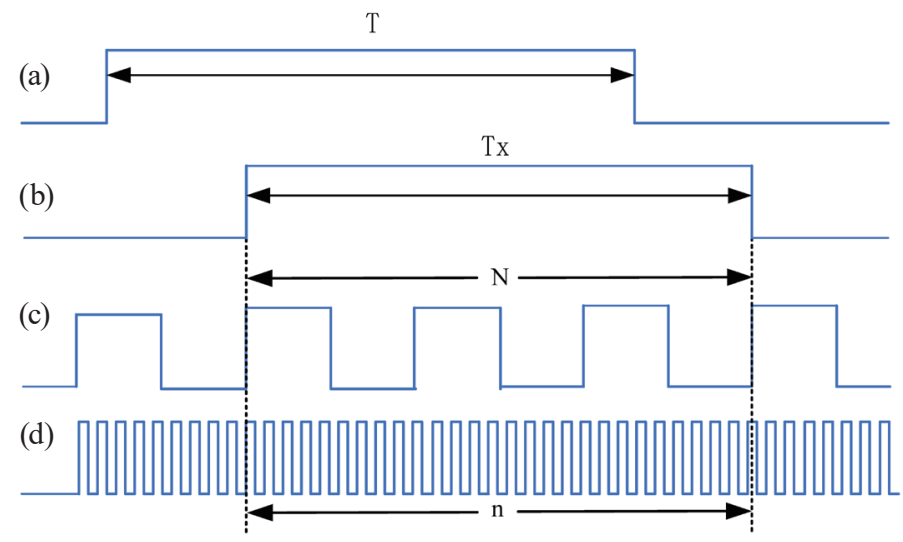

Fig. 13. (Color online) Schematic of frequency measurement.

$$
f_{s}=\frac{N}{n} f_{0},
$$

where $n$ is the number of cycles of the reference signal and $f_{0}$ is the frequency of the reference signal.

\section{Sensitivity Estimation Method}

Earth's magnetic field slowly changes with time owing to natural factors called diurnal variation. The magnetometer sensor also receives EMI components due to the influence of the human environment. In addition, the sensor contains the noise of the instrument caused by the uncertainty of the frequency counter. The measured magnetic field can be expressed as follows in terms of these three factors:

$$
B=B_{0}+B^{\prime}+B^{\prime \prime}
$$

where $B_{0}$ is Earth's magnetic field; $B^{\prime}$ comprises low-frequency components, such as diurnal variation and drift, and high-frequency components of EMI noise; and $B^{\prime \prime}$ is the reading noise of the PPM. The goal is to accurately estimate the noise level of $B^{\prime \prime}$, namely the sensitivity. 


\subsection{Direct estimation method}

Equation (7) indicates that if $B$ can be measured in the environment without $B^{\prime}$, or the highfrequency component of $B^{\prime}$ is substantially smaller than $B^{\prime \prime}$, then the reading noise level or sensitivity $B^{\prime \prime}$ can be estimated using the following equation:

$$
\sigma=\sqrt{\frac{1}{N-1} \sum_{i=1}^{N}\left(B_{i}-\bar{B}\right)^{2}},
$$

where $B_{i}$ is the magnetic field measured at different times and $\bar{B}$ is the mean value of $B_{i}$.

\subsection{Fourth-order difference}

However, obtaining environments without $B^{\prime}$ is difficult. Low-frequency components such as diurnal variation are inevitable when a single instrument is measured in a natural environment. The low-frequency components $B^{\prime}$ can be effectively filtered out by the fourth-order difference method, and an accurate sensitivity estimation can be obtained. The sensitivity of a single instrument obtained by the fourth-order difference method is estimated as

$$
\sigma=\frac{1}{\sqrt{70}} \sqrt{\frac{1}{n-1} \sum_{i=1}^{n}\left(T_{i}-\bar{T}\right)^{2}},
$$

where $T_{i}=B_{i-2}-4 B_{i-1}+6 B_{i}-4 B_{i+1}+B_{i+2}$ is the fourth-order difference function for the measured data, $\bar{T}$ is the mean value of $T_{i}$, and $n$ is the number of magnetic field values involved in the statistics. The fourth-order difference function has a high-pass characteristic, which can filter out the low-frequency and DC components in Earth's magnetic field.

\subsection{Synchronization method}

The synchronization method refers to the STD of the difference between the magnetic field values measured by two simultaneous measurement instruments as an estimate of the sensitivity of a single instrument. Assuming that the sensitivities of the two instruments are the same, and $B_{0}$ and $B^{\prime}$ are removed from the total magnetic field after differentiating the two synchronized data, the energy of the noise $B^{\prime \prime}$ is doubled. Thus, the sensitivity can be obtained as

$$
\sigma=\frac{1}{\sqrt{2}} \sqrt{\frac{1}{n-1} \sum_{i=1}^{n}\left(X_{i}-\bar{X}\right)^{2}},
$$

where $X_{i}=B_{1 i}-B_{2 i}, \bar{X}$ is the mean value of $X_{i}$, and $B_{1 i}$ and $B_{2 i}$ are data measured by two synchronous magnetometers. 


\section{Experimental Results}

Typical noisy and quiet electromagnetic environments were selected with a cycle rate of $5 \mathrm{~s}$ to evaluate system sensitivity and investigate the influence of EMI on sensitivity evaluation. The synchronous error between magnetometers JPM-4 \#001 and JPM-4 \#002 is less than $1 \mathrm{~ms}$.

\subsection{Synchronization sensitivity estimation under quiet environment}

We performed an observation of the diurnal variations of Earth's magnetic field for nearly $10 \mathrm{~h}$ about $5 \mathrm{~km}$ south of Dalin Town, Tongliao District, Inner Mongolia on October 22, 2019, to estimate the sensitivity of the magnetometers. Figure 14 shows the diurnal variation of the magnetic field observed by the two magnetometers, the fourth-order difference of the magnetic fields of JPM-4 \#001 and JPM-4 \#002, and the value of the fourth-order difference.

Figure 14(a) shows that the observed magnetic field of Earth fluctuates between 55230 and $55245 \mathrm{nT}$, in which accidental magnetic sparks are found. The two instruments consistently

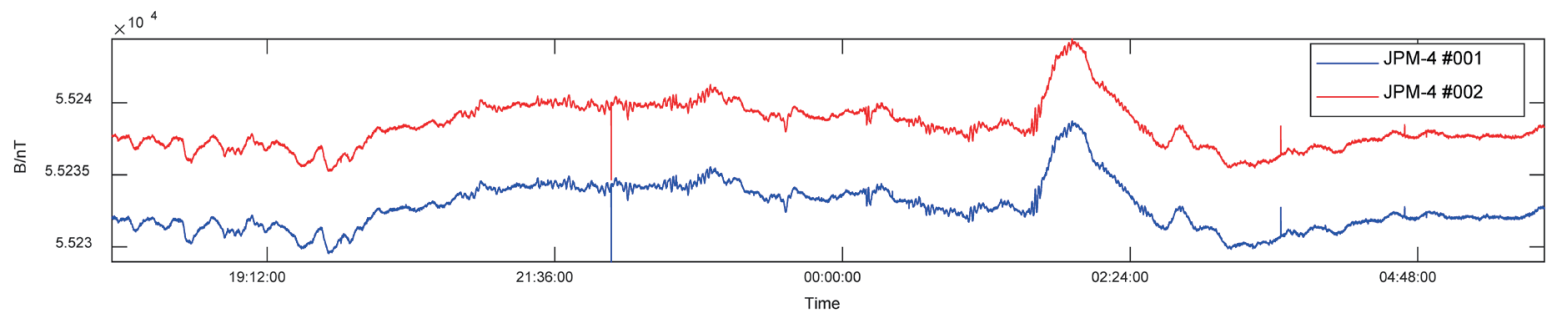

(a)

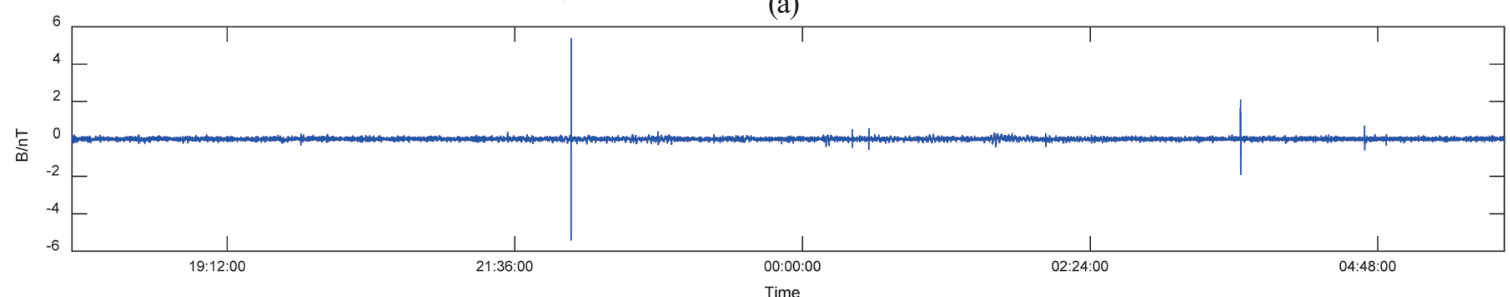

(b)

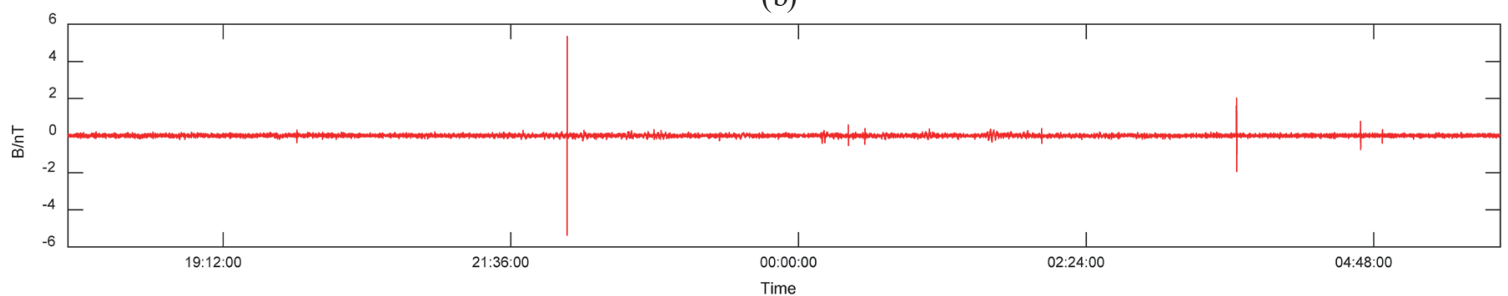

(c)

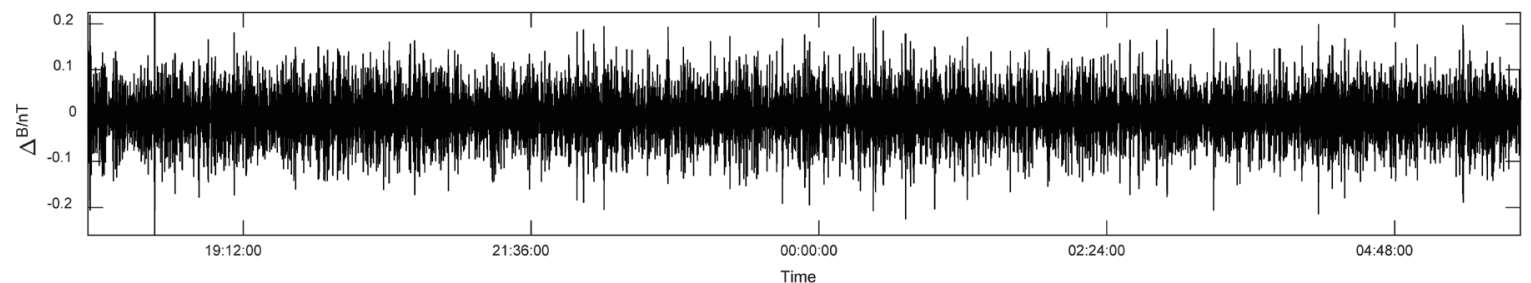

(d)

Fig. 14. (Color online) Synchronous measurement results of JPM-4 \#001 and \#002 (5 s cycling rate). 
record the diurnal changes in Earth's magnetic field. Figures 14(b) and 14(c) reveal that the fourth-order difference of the two magnetic field values eliminates the diurnal variation. The fourth-order differential peaks of the two sets of magnetic fields are both less than $1.0 \mathrm{nT}$ after ignoring the interference of Earth's magnetic field sparks, indicating that the external EMI highfrequency noise is low. The calculated STDs are 0.0775 and $0.0759 \mathrm{nT}$, and the results are approximately equal. This result indicates that the consistency of the instruments is very high and can meet the preconditions for the sensitivity estimation of the synchronization method. Figure 14(d) again shows the variations in the two fourth-order differences used to estimate the target sensitivity accurately. From Eq. (10), the estimated sensitivity is $0.0419 \mathrm{nT}$, which is better than that of $0.1 \mathrm{~T}$ for most commercial magnetometers.

\subsection{Synchronization sensitivity estimation under noisy environment}

Measurements were implemented on the campus of Jilin University to verify the performance of the synchronization sensitivity estimation method in a noisy environment, and the results are shown in Fig. 15.

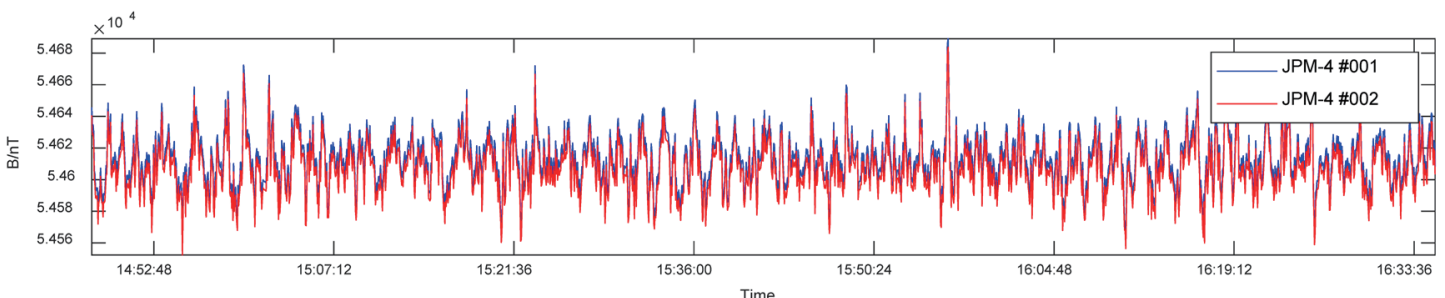

(a)

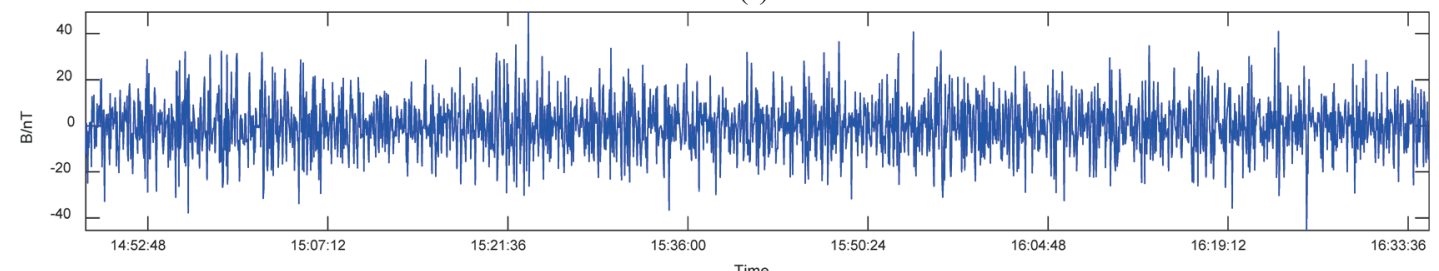

(b)

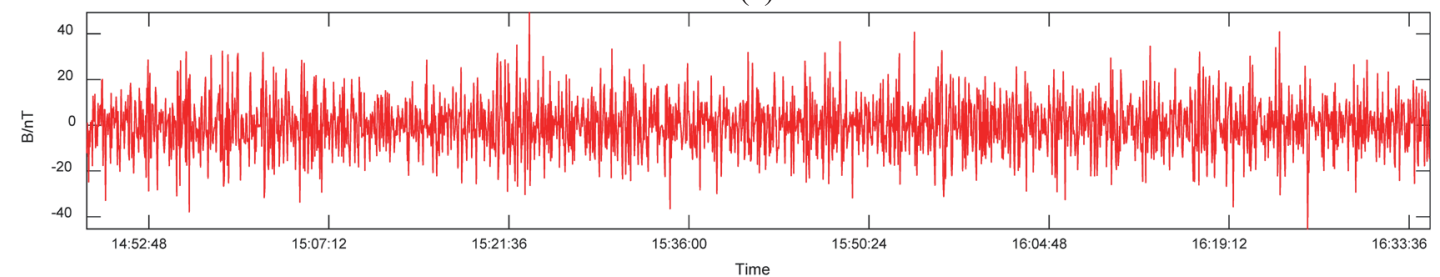

(c)

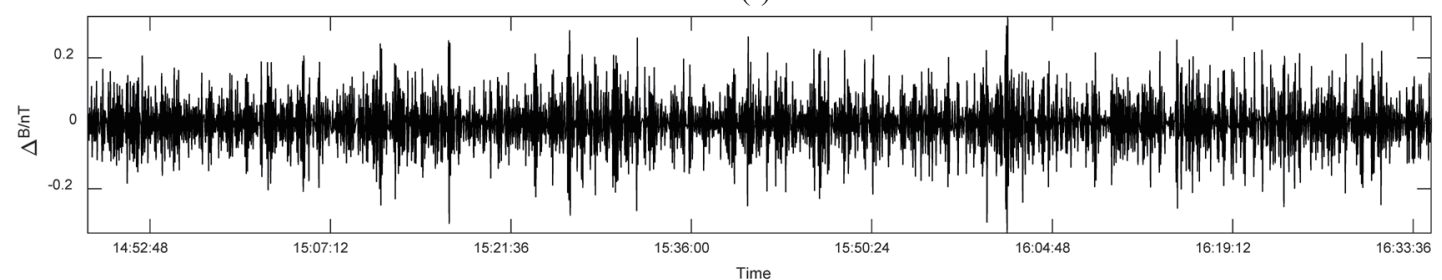

(d)

Fig. 15. (Color online) Synchronous measurement results of JPM-4 \#001 and \#002 (3 s cycling rate). 
Figure 15(a) shows that the magnetic field is consistently recorded by the two instruments. The data after fourth-order difference processing for JPM-4 \#001 and JPM-4 \#002 are shown in Figs. 15(b) and 15(c), and the calculated STDs are 6.405 and $6.394 \mathrm{nT}$, respectively. The STDs of nT order indicate that the EMI noise $B^{\prime}$ is substantially larger than the reading noise of the PPM $B^{\prime \prime}$. Thus, STD can be used as an indicator of the electromagnetic environment noise level. Figure 15(d) presents the differential result from the data in Figs. 14(b) and 14(c). The sensitivity is estimated to be $0.064 \mathrm{nT}$ from Eq. (10) with the elimination of the external interference. This value is larger than that estimated in the quiet electromagnetic environment.

The comparison between Sects. 5.1 and 5.2 shows that the sensitivity estimated in a quiet environment is more precise than that in a noisy environment. The EMI noise has a substantial impact on reading noise in noisy environments, which affects the accuracy of the synchronous sensitivity estimation method.

\section{Conclusion}

We adopted a compact 8 type coil for the JPM-4 proton magnetometer sensor, which has good omnidirectivity. The coil resistance is $18 \Omega$, the polarization current is $0.67 \mathrm{~A}$, and the polarization power is $8.0 \mathrm{~W}$. The SNR of the FID signal can reach 31/1 and the decay constant of the FID signal is approximately $0.95 \mathrm{~s}$. The JPM-4 proton magnetometer has a synchronization function with a synchronization error of less than $1 \mathrm{~ms}$.

The DC and low-frequency components in the total field can be filtered out using a fourthorder difference. The STDs of a single instrument with the fourth-order difference were estimated to be 0.078 and $6.4 \mathrm{nT}$ in quiet and noisy environments, respectively, indicating its ability to characterize the environmental noise level. The EMI in the total field can be effectively eliminated with the synchronous method. The sensitivities of two JPM-4 magnetometers calculated from synchronous data were 0.064 and $0.042 \mathrm{nT}$ in noisy and quiet environments, respectively. Thus, the synchronous method can effectively eliminate the EMI components and accurately evaluate the sensitivity of the proton magnetometer, and the estimated sensitivity was $0.04 \mathrm{nT}$.

The results of synchronous sensitivity estimation indicate that the JPM-4 proton magnetometer developed by Jilin University can measure Earth's magnetic field with sufficient sensitivity for the observation of diurnal variations and can be used for other applications involving the measurement of weak magnetic fields. The synchronization method given in this article is also applicable to the sensitivity estimation of the optical pump magnetometer.

\section{Acknowledgments}

This work was supported by the National Natural Science Foundation of China under Grant No. 61771218. 


\section{References}

1 E. M. Purcell, H. C. Torrey, and R. V. Pound: Phys. Rev. 69 (1946) 37. https://doi.org/10.1103/PhysRev.69.37

2 F. Bloch: Physica 17 (1950) 460. https://doi.org/10.1103/PhysRev.70.460

3 R. H. Varian: US, US2561490 AP. 1951. https://www.freepatentsonline.com/2561490

4 G. S. Waters: Nature 176 (1955) 691. https://doi.org/10.1038/176691a0

5 G. S. Waters and G. Phillips: Geophys. Prospect. 4 (1956) 1. https://doi.org/10.1111/j.1365-2478.1956.tb01392.x

6 A W. Overhauser: J. Phys. Rev. 92 (1953) 411. https://doi.org/10.1103/PhysRev.92.411

7 A. Abragam: J. Phys. Rev. 98 (1955) 1729. https://doi.org/10.1103/PhysRev.98.1729

8 G. Breit and I. I. Rabi: J. Special Studies Papers 38 (1931) 2082. https://doi.org/10.1103/PhysRev.38.2082.2

9 I. Solomon: J. Phys. Rev. 99 (1955) 559. https://doi.org/10.1103/PhysRev.99.559

10 J. A. Koehler: Comox Canada (November 2004). https://www.do-cin.com/p-747185843.html

11 N. Kernevez and H. Glenat: IEEE Trans. Magn. 27 (2002) 5402. https://doi.org/10.1109/20.278852

12 D. Duret, J. Bonzom, M. Brochier, M. Frances, J. M. Leger, R. Odru, C. Salvi, T. Thomas, and A. Perret: IEEE Trans. Magn. 31 (1995) 3197. https://doi.org/10.1109/20.490326

13 I. Hrvoic: Proc. Int. Workshop on Geomagnetic Observatory Data Acquisition and Processing (1990). http:// www.geophysik.uni-bremen.de/statisch/downloads/254/Overhauser-Magnetometers (accessed April 2021).

14 J. Lenz and S. Edelstein: IEEE Sens. J. 6 (2006) 631. https://doi.org/10.1109/JSEN.2006.874493

15 P. Mahavarkar, S. Singh, S. Labde, V. Dongre, and A. Patil.: J. Instrum. 12 (2017) T05002. https://iopscience. iop.org/article/10.1088/1748-0221/12/05/T05002

16 H. Dong, H. Liu, J. Ge, Z. Yuan, and Z. Zhao: Sensors 16 (2016) 510. https://doi.org/-10.1109/tim.2016.2516299

17 H. Liu, H. Dong, Z. Liu, J. Ge, B. Bai, and C. Zhang: J. Instrum. (2017) https://iopscience.iop.org/ article/10.1088/17480221/12/1-0/T10008 (accessed May 2021).

18 H. Dong, H. Liu, J. Ge, Z. Yuan, and Z. Zhao: IEEE Trans. Instrum. Meas. 65 (2016) 898. https://doi. org/10.1109/TIM.2016.2516299

19 C. Tan, J. Wang, and Z. Li: Measurement (2019). https://doi.org/10.1-016/j.measurement.2018.10.016 